\title{
Distinct Neuroanatomical Structures of Acupoints Kidney 1 to Kidney 8: A Cadaveric Study
}

\author{
Melissa Lee, ND, RAc, ${ }^{1}$ Ryan Longenecker, RAc, ${ }^{2}$ Samuel Lo, RAc, ${ }^{3}$ and Poney Chiang, PhD, RAc ${ }^{4}$
}

\begin{abstract}
Objective: The Systematic Classic/Zhen Jiu Jia Yi Jing (ZJJYJ, 鍼灭甲乙經) is considered to be the first complete acupuncture manual to detail the location and meridian assignations of 349 acupuncture points. Despite numerous transcriptions and editing changes, many traditional acupuncturists adhere to the classics and rarely question their validity. However, ushering the use of acupuncture into the modern era requires examining acupuncture point locations objectively by comparing contemporary anatomical knowledge with classical texts. The aim of this research was to examine distinct neuroanatomical targets associated with acupuncture points to: (1) standardize the precise neuroanatomical target of each acupuncture point; and (2) crossreference neuroanatomical targets with classical point locations. This was done to demonstrate ancient authors' intentions when describing acupuncture points as coordinates used to stimulate the peripheral nervous system.

Materials and Methods: The unique neuroanatomical targets associated with acupuncture points on the Foot Shao Yin Kidney meridian were defined. Specifically, KI 1 through KI 8 were examined by comparing classical point locations from the ZJJYJ with modern standardized textbook locations from Chinese Acupuncture and Moxibustion, current anatomical literature, the current authors' cadaver dissection research, and electrostimulation of acupuncture points in healthy volunteers.

Results: KI 1-KI 8 correlated with motor entry points as well as with nerve branches and vessels derived from the posterior tibial neurovascular bundle.

Conclusions: This research demonstrated a procedure to verify and standardize the distinct neuroanatomical structures of acupuncture points. Standardization of neuroanatomical targets of acupuncture points will enable researchers and clinicians to obtain reproducible results in clinical treatments and research protocols.
\end{abstract}

Keywords: electroacupuncture, Traditional Chinese Medicine, meridians, Kidney meridian, neuroanatomy

\section{INTRODUCTION}

$\mathbf{F}$ OR THOUSANDS OF YEARS, the art and science of acupuncture has relied on the descriptions of meridians to locate acupuncture points and describe their functions. Medical classics such as the Plain Question ( $\mathrm{Su}$ Wen, SW,
素問) ${ }^{1}$ and Spiritual Pivot (Ling Shu, LS, 灵枢经) $)^{2}$ theorized that meridians were conduits that transport Qi (氣), or energy, which is widely believed to have no direct correlation with any known anatomical structures. Popular opinion suggests that, due to conservative Confucian values and ancestral worship, the ancient Chinese did not dissect their

\footnotetext{
${ }^{1}$ River Clinic, Toronto, Ontario, Canada.

${ }^{2}$ Avenue Acupuncture, Toronto, Ontario, Canada.

${ }^{3}$ Toronto Acupuncture \& Osteopathy Clinic, Toronto, Ontario, Canada.

${ }^{4}$ Faculty of Health, School of Kinesiology and Health Sciences, Norman Bethune College, York University, Toronto, Ontario, Canada.
}

CME available online at www.medicalacupuncture.org/cme Questions on page 27. 
dead. Rather, meridians and acupuncture-point locations were inferred through the use of palpation, meditation, and exercise techniques used on living patients. However, a growing body of scientific literature suggests that meridians and acupuncture points were not discovered through subjective inferences but were based instead on observations of cadaveric dissections, and that, therefore, acupuncture was firmly based on the science of anatomy. ${ }^{2,3}$

Pioneering research to investigate the anatomical significance of acupoints systematically began in 1959 at the Shanghai First Medical College (now the Shanghai Medical College of Fudan University). ${ }^{4}$ Researchers demonstrated that 323 of 324 dissected acupoints were associated with peripheral nerves; 93.8\% (304/324 acupoints) were associated with superficial cutaneous nerves; and 52.5\% (170/324 acupoints) were associated with deep nerve trunks. ${ }^{5}$ Similarly, researchers at the Shanghai Traditional Chinese Medicine (TCM) College (now Shanghai TCM University) found that, among 309 dissected acupoints, needles touched the trunks of nerves directly in 152 acupoints (49.2\%). The nerve trunks were within $0.5 \mathrm{~cm}$ of the remaining $157 \mathrm{acu}-$ points $(50.8 \%)$ acupoints. ${ }^{5}$ These studies provided compelling insight into what ancient acupuncturists were observing and documenting when they chose to record-in what would eventually become seminal medical canonsthe precise location of acupuncture points as unique peripheral nerve-stimulation sites.

The Systematic Classic/Zhen Jiu Jia Yi Jing (ZJJYJ, 鍼尒甲乙經), written in $259 \mathrm{AD}$, is considered to be the first complete acupuncture manual to detail the location and meridian assignations of 349 acupuncture points. ${ }^{6}$ Despite numerous transcriptions and editing changes more than 1700 years ago, many traditional acupuncturists adhere to the classics and rarely question their validity in modern medicine. It is the authors' joint opinion that, to usher the use of acupuncture into the modern era truly, acupuncture-point locations need to be examined objectively by comparing contemporary anatomical knowledge with classical textual research.

This would achieve two goals. The first goal would be standardization of the precise neuroanatomical target of each acupuncture point. If point stimulation is solely based on a two-dimensional surface coordinate without defining its three-dimensional neuroanatomical target beneath the skin, different researchers stimulating the same acupuncture point could, in fact, be stimulating different structures. This makes interpretation of outcomes from different studies impossible. Standardizing the precise target of each acupuncture point would allow for reproducibility of results in research and clinical settings. The second goal would be, using classical point locations to crossreference distinct neuroanatomical targets, to demonstrate to traditional acupuncturists the ancient sages' original intentions in using precise locations to stimulate the peripheral nervous system. Overall, this would, it is hoped, inspire interest in studying neuroanatomy so that interprofessional collaboration and communication based on the same medical language would become possible.

With these goals in mind, this article reports on the examination of distinct neuroanatomical targets associated with acupuncture points on the Foot Shao Yin Kidney meridian, specifically Kidney1 (KI 1) through Kidney 8 (KI 8).

\section{MATERIALS AND METHODS}

The distinct neuroanatomical target associated with each acupuncture point, KI 1 through KI 8, was examined by consulting Chinese Acupuncture and Moxibustion ${ }^{7}$ and ZJJYJ texts. This information was compared with modern anatomical resources/research articles, the current authors' own cadaveric dissections, and electrostimulation of acupoints in healthy volunteers. When the Chinese Acupuncture and Moxibustion ${ }^{7}$ and ZJJYJ texts had different locations for these points, they were verified with peerreviewed articles, cadaveric dissections, and monopolar electrostimulation of the acupoints on healthy volunteers.

The 349 acupoints listed in the ZJJYJ were transliterated into English as the English version is a translation itself. Transliteration reduces misinterpretation of anatomical landmarks that are required for precise locations. This article includes the transliterations of KI 1 through KI 8 (Table 1).

\section{Anatomical Dissection}

The dissection specimen was an 88-year-old female body acquired for anatomical education via the Human Body Donation Program at Queen's University, in Kingston, Ontario, Canada. The specimen was fixed with vascular perfusion, using a mixture of liquid phenol, 95\% ethanol, glycerin, and water. The dissection occurred at the Anatomy Learning Centre in the Queen's University as a part of its Complementary and Alternative Medicine-Human Dissection Experience program.

\section{Electrostimulation of Acupoints}

The neuroanatomical targets of KI 1 through KI 8 were verified by electroacupuncture. Stimulation of KI 1 through KI 8 was performed with a Pointer Plus device (a $10-\mathrm{Hz}$ monopolar electrostimulation device; Mayfair Medical Supplies Ltd., Hong Kong). The responses, which were cutaneous paresthesia and/or muscle recruitment, were then compared to the actions of the examined cutaneous nerves, muscles, and motor nerves.

\section{RESULTS}

The results were as follows:

KI 1,Gushing Spring-KI-1 coincided with the location of the deep branch of the lateral plantar nerve (Fig. 1). 


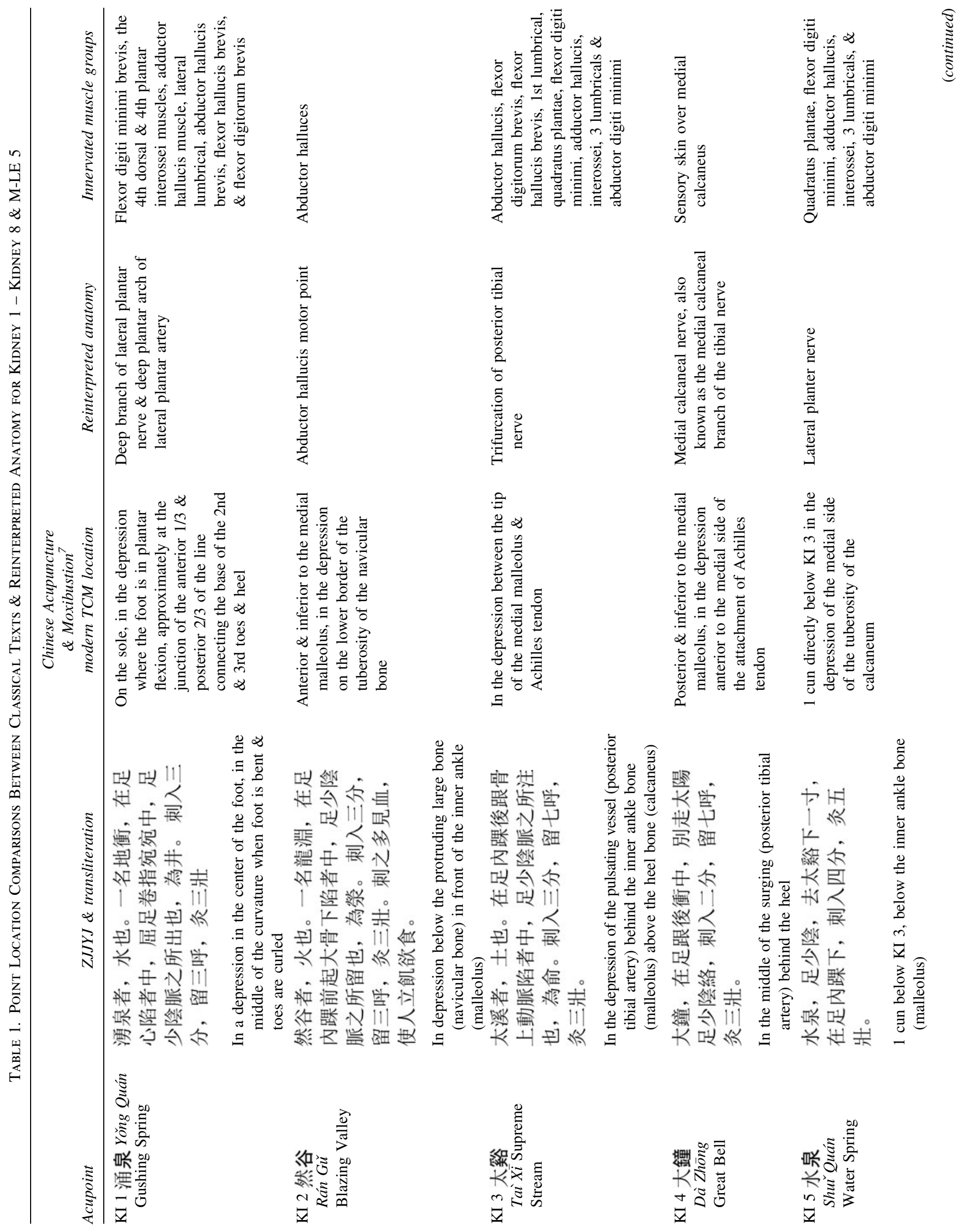




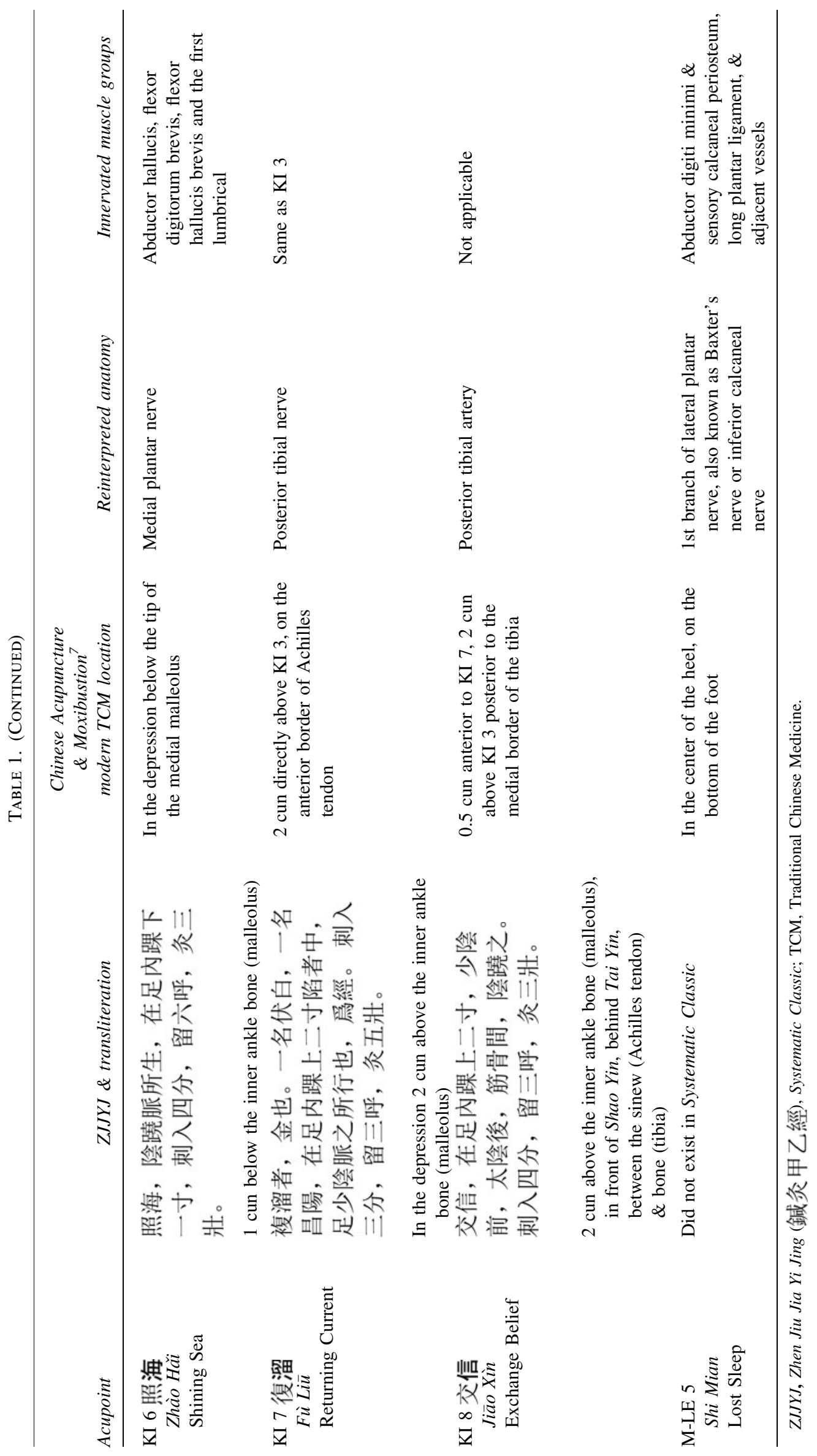




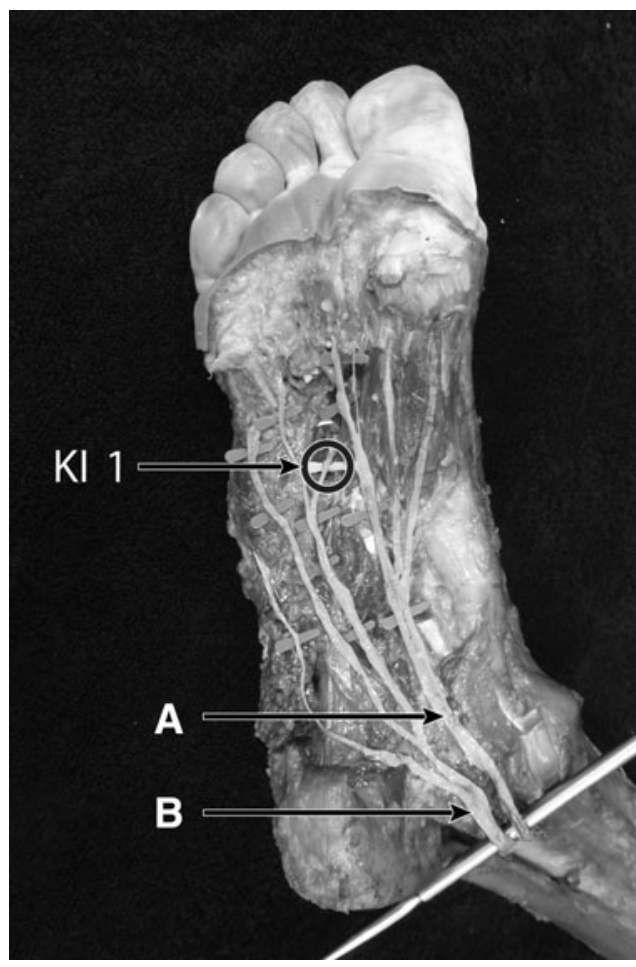

FIG. 1. KI 1 at the deep branch of lateral plantar nerve. (A) Medial plantar nerve. (B) Lateral plantar nerve.

KI 2, Blazing Valley_-"In the depression below the bone" refers to the motor point for the abductor hallucis muscle (Fig. 2). Supplementary Video 1 demonstrates that needling of this point elicited abduction of the hallux. KI 3, Supreme Stream-The "pulsating vessel" likely refers to the posterior tibial artery (Fig. 3). The posterior tibial nerve lies adjacent and posterior to the artery. This point is located at the trifurcation of the posterior tibial

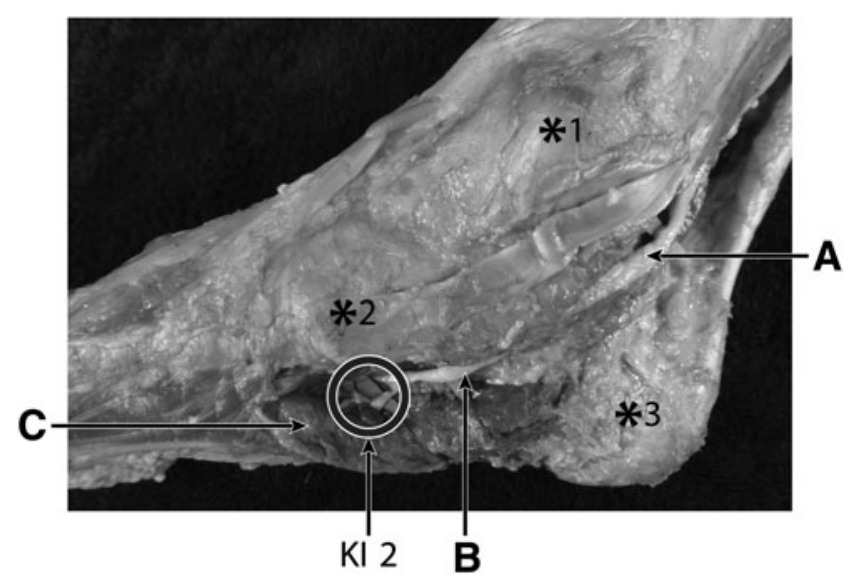

FIG. 2. KI 2 at the motor point for the abductor hallucis. (A) is the posterior tibial nerve; (B) is the medial plantar nerve; and (C) is the abductor hallucis muscle. $* 1$, medial malleolus; $* 2$, navicular bone; $* 3$, calcaneus bone.

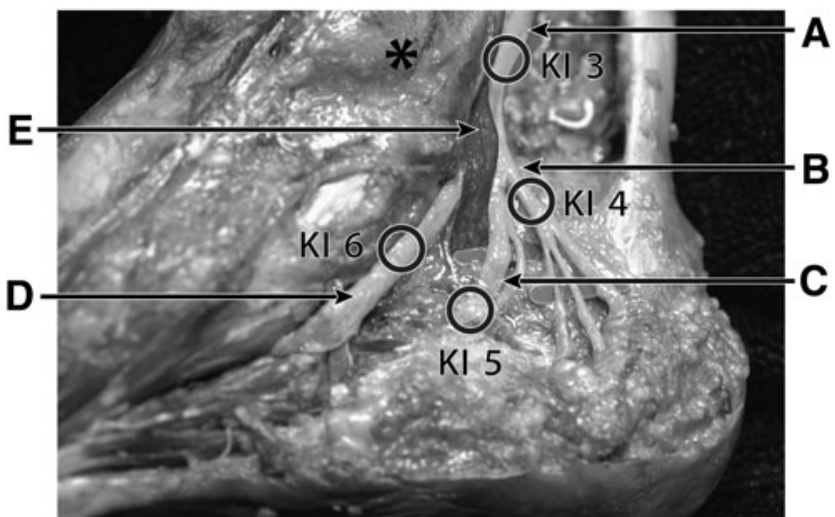

FIG. 3. Branching of the posterior tibial nerve. KI 3 as (A), posterior tibial nerve; KI 4 as (B), medial calcaneal branch; KI 5 as (C), lateral plantar nerve; and KI 6 as (D), medial plantar nerve and (E) posterior tibial artery.*Medial malleolus.

nerve into the medial calcaneal, medial, and lateral plantar branches. Electrostimulation of KI 3 resulted in flexion of the medial and lateral parts of the foot, attributed to the medial and lateral plantar nerves that are derived from the trifurcation of the posterior tibial nerve. See Supplementary Video 2.

KI 4, Great Bell-Dissection of this area revealed the presence of the medial calcaneal branch of the tibial nerve, anterior to the Achilles tendon (Fig. 3). Needling of KI 4 resulted in paresthesia in the medial calcaneus.

KI 5, Water Spring-KI 5 lies on the lateral plantar nerve (Fig. 3). Stimulation of KI 5 along the lateral plantar nerve elicited flexion of the 5th toe via the flexor digiti quinti brevis (Supplementary Video 2) and abduction of the 5 th toe via the abductor digiti minimi (Supplementary Video 3).

KI 6, Shining Sea-The medial plantar nerve is the superior branch originating from the posterior tibial nerve (Fig. 3). This branch innervates the abductor hallucis, flexor digitorum brevis, flexor hallucis brevis, and the first lumbrical. Stimulation of KI 6 resulted in flexion of the 1st toe, which coincides with the medial plantar nerve (Supplementary Videos 2 and 4). Abduction of the 1st first toe is not shown.

KI 7, Returning Current-Dissection revealed the presence of the posterior tibial nerve (Fig. 4).

KI 8, Exchange Belief-This point corresponded to the posterior tibial artery, which lies anterior to the posterior tibial nerve (Fig. 4).

Together KI 7 and KI 8 corresponded to the posterior tibial neurovascular bundle.

Please see Table 1 for a summary of acupuncture-point locations according to the classic texts and compared with modern-day neuroanatomy. Refer to Figures 1-4 and Supplementary Videos 1-4. The videos demonstrate electrostimulation of KI 2, KI 3, KI 5, and KI 6. 


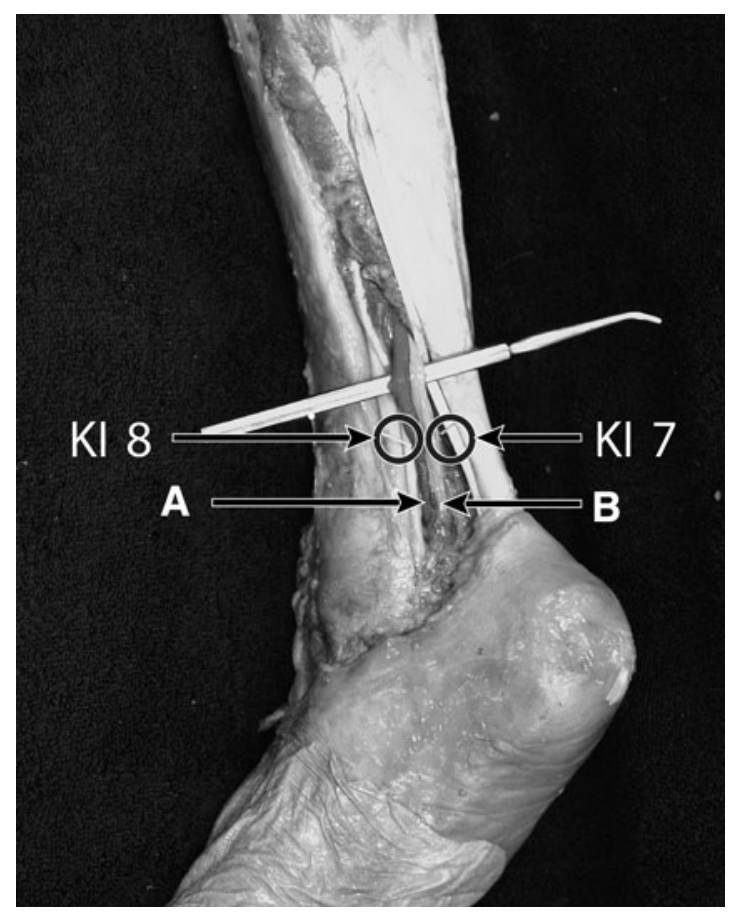

FIG. 4. Posterior tibial neurovascular bundle. (A) Posterior tibial artery. (B) Posterior tibial nerve.

\section{DISCUSSION}

\section{KI 1 and KI 2}

KI 1 coincides with the deep branch of the lateral plantar nerve (Fig 1). The deep branch of the lateral plantar nerve consists of nerve fascicles of muscular branches to the flexor digiti quinti brevis muscle, the 4th dorsal and 4th plantar interossei muscles, and the adductor hallucis muscle, as well as to the articular branches to the 5th metatarsophalangeal joint. More specifically, the deep branch of the lateral plantar nerve supplies most of the intrinsic and short muscles of the sole of the foot: lateral lumbrical; abductor hallucis brevis; flexor hallucis brevis; and flexor digitorum brevis, collectively known as the $L A F F$ muscles. ${ }^{8}$ The muscular branch to the oblique head of the adductor hallucis separates directly from the nerve fascicles constituting the deep branch of the lateral plantar nerve, whereas the muscular branch to the transverse head arises from the common branch innervating other intrinsic muscles of the foot. ${ }^{8}$ Dissections performed by Shaw similarly showed a correlation with the Chinese Acupuncture and Moxibustion ${ }^{7}$ locations of KI 1 and the corresponding anatomical structure of the deep plantar artery. ${ }^{9}$

KI 2 corresponds to the motor point of the abductor hallucis muscle, which is located more inferiorly and posteriorly to the navicular. ${ }^{10,11}$ This muscle is an intrinsic pedal muscle that plays an important role in supporting the medial longitudinal arch and controlling pronation during a static stance. ${ }^{12}$ Specifically, abductor hallucis fatigue (stimulated by fatiguing exercises of the foot intrinsic muscles) or the administration of a tibial nerve block decreases abductor hallucis electromyographic activity and increases navicular drop. ${ }^{12,13}$ Navicular drop can cause changes in the arch that lead to foot and plantar problems. Finally, muscle imbalance between adductor hallucis and abductor hallucis is apparent in hallucis valgus deformity. ${ }^{14}$

Together, KI 1 corresponds to the deep branch of the lateral plantar nerve, which also innervates the adductor hallucis muscle, while KI 2 corresponds to abductor hallucis motor point. These two acupuncture points could be implicated in the treatment of foot-related problems that affect the LAFF muscles.

\section{KI 3 through KI 6}

KI 3, KI 4, KI 5, and KI 6 all correspond to aspects of the tibial nerve. The tibial nerve provides plantar sensation to the entire foot. The tibial nerve ramification occurs posteriorly to the medial malleolus, inside the tarsal tunnel. ${ }^{15}$ The medial calcaneal branch separates from the tibial nerve above the flexor retinaculum but is not higher than the medial malleolus (Fig. 3).

KI 4 is located on the medial calcaneal branch, which provides sensation to the heel pad. Transection of the calcaneal branch results in numbness of the heel pad. ${ }^{16}$ Likewise, needling of KI 4 results in paresthesia in the medial calcaneus. One interesting observation to note is that $\mathrm{KI} 4$ is also a Luo-Connecting point of the channel, which is said to encircle the heel and connect with the Bladder (BL) channel. The medial calcaneus nerve anastomoses with the lateral calcaneal nerve (sural), which satisfies this KI-to-BL connection.

Targeting this anatomy therapeutically can be useful for foot-related conditions. For example, the medial tarsal tunnel contains the following structures: flexor retinaculum (tunnel roof); talus and calcaneus (tunnel floor); tendons of tibialis posterior; flexor digitorum longus; posterior tibial artery; posterior tibial nerve; and flexor hallucis longus (tunnel content).

Medial tarsal tunnel syndrome (MTTS) is a condition that presents as pain or paresthesia along the medial plantar nerve or lateral plantar nerve (or both) sensory distribution from the ankle to the sole, with a positive Tinel's sign over the flexor retinaculum, and abductor hallucis and abductor digit quinti muscle weakness in later presentations. ${ }^{17,18} \mathrm{In}$ terestingly, one of the most-sensitive methods in differentially diagnosing MTTS is measurement of the sensory conduction velocity of the medial and lateral plantar nerves. This is done by stimulating the nerves at the vault of the foot. ${ }^{17}$ It would be valuable to explore the use of KI 3-to-KI 6 acupoints to stimulate the posterior tibial nerve, its branches (e.g., the medial and lateral plantar nerves), and surrounding foot structures as a potential diagnostic and 
treatment option for MTTS. This could be used adjunctively with conservative methods-such as taping, bracing, chiropractic adjustment, massage, or fascial strippingultimately, to relieve irritation and decompress the nerve.

Treatment of relevant anatomy might also be applicable for Baxter's nerve-entrapment issues. Baxter's nerve entrapment can give rise to precalcaneal pain on the heel, which can also be affected in MTTS. Often, this syndrome mimics signs of plantar fasciitis and differential diagnosis in a case of plantar fasciitis is important. In Baxter's nerve entrapment, it is not the medial calcaneal branch (as it separates before the flexor retinaculum) but the first branch of the lateral plantar nerve (inferior calcaneal nerve) that is compressed. ${ }^{19} \mathrm{KI} 5$, which corresponds to the lateral plantar nerve, could be indicated for Baxter's nerve entrapment. The Extra point M-LE 5; Lost Sleep or Shi Mian (Table 1) corresponds to the inferior calcaneal nerve (Baxter's nerve).

\section{KI 7 and KI 8}

KI 7. In the TCM context, KI 7 is known as a strong empirical point for balancing sweating. ${ }^{7}$ Sweating is a sudomotor response attributed to the autonomic nervous system (ANS). Anatomically, KI 7 corresponds to the tibial nerve and is located at the level of the ankle. At the level of the ankle, the tibial nerve, small arteries around the tibial nerve, and the calcaneal tendon all have sympathetic fibers. $^{20,21}$ Taken together, the sympathetic fibers in the tibial nerve likely innervate the paratendinous tissue as well as the paratendinous microvasculature of the calcaneal tendon. This could potentially explain the ability of KI 7 to reduce or stop sweating.

Another example of how the tibial nerve has been used to treat an ANS-related condition, is in overactive bladder $(\mathrm{OAB})$ syndrome. The posterior tibial nerve is a mixed sensory-motor nerve with axons that pass through L-4-S-3 spinal nerve roots. These nerve roots have been known to contain peripheral nerves that are involved in sensory and motor control of the bladder and pelvic floor. These nerve roots are also targets utilized by urologists for percutaneous tibial nerve stimulation treatment. Stimulation inhibits bladder activity by stimulating large diameter somatic afferent fibers. This evokes a central inhibition of the micturition reflex pathway in the spinal cord or brain. ${ }^{22}$ More specifically, Tai et al. were also able to isolate the effect of precise frequencies of posterior tibial nerve stimulation in a cat model: $5-\mathrm{Hz}$ and $30-\mathrm{Hz}$ stimulation of the posterior tibial nerve inhibited bladder activity; and short-duration stimulation induced a persistent poststimulation inhibitory effect and increased bladder capacity. ${ }^{23}$

Given that KI 7 corresponds to the tibial nerve, which has been used to treat syndromes associated with the ANS, such as the $\mathrm{OAB}$ syndrome, further investigation of the use of KI 7 and its relation to the autonomic nervous system is warranted.
KI-8. KI 8 is the $X i$-Cleft point of the Yin Motility vessel. This point is the intersection of the Yin Motility and the Foot Shao Yin Kidney channel. Xi-Cleft points are related to Blood, while the Vessel/Mai in the Yin Motility all hint at a blood-vessel-related anatomy and function. For these reasons, the current authors suggest strongly that the descriptions in the classic texts are referring to the posterior tibial artery (as opposed to posterior tibial nerve), which lies anteriorly to the nerve.

\section{CONCLUSIONS}

Based on literature reviews of classical texts, anatomical literature, and the current authors' dissections and electrostimulation videos, distinct neuroanatomical targets were demonstrated for the acupuncture points KI 1 to KI 8 . These anatomical targets include the tibial nerve trunk, the tibial nerve branches, and the muscles that the branches innervate, as well as the tibial artery. Identifying these targets will allow for standardization of acupuncture points and reproducibility in clinical treatments and research protocols.

Clinically, the current authors also found that the neuroanatomical structures of KI 1 to KI 8 have been indicated in the treatment of certain entrapment syndromes of the foot (e.g., MTTS) and syndromes involving the ANS (e.g., OAB syndrome). Taken together, future studies should explore the use of the Kidney channel clinically as an adjunct treatment or for similar applications.

Finally, the current authors hope that this approach will inspire acupuncturists' interest in studying neuroanatomy so that interprofessional collaboration based on the same medical language is made possible. It will ultimately be in patients' best interest for their healthcare providers to communicate effectively with each other.

\section{ACKNOWLEDGMENTS}

The authors acknowledge Chris Gordon, DC, a colleague who assisted in the development of this work. Also Craig Harness RMT, DO(Q), of Queen's University, Canada, is acknowledged for his assistance with the cadaver dissections.

\section{AUTHOR DISCLOSURE STATEMENT}

No competing financial interests exist.

\section{REFERENCES}

1. The Plain Question Chinese Text Project. Online document at: www.ctext.org/huangdi-neijing/suwen Accessed Sept 10, 2018. 
2. Li Z, Liu Z. The Yellow Emperor's Canon of Medicine: Spiritual Pivot I (Library of Chinese Classics), 1st ed. Xi' an, China: World Publishing Cooperation; 2008.

3. Chiang P. What is the point of acupuncture? Med Acupunct. 2015;27(2):67-80.

4. Li D. The pathway of the twelve regular meridians and anatomical observation on points and human structure. Shanghai College of Traditional Chinese Medicine 1960; 8(1): 57-85.

5. Zhou F, Huang D, Xia Y. Neuroanatomic basis of acupuncture points. In: Xia Y, Cao X, Wu G, Cheng J, eds. Acupuncture Therapy for Neurological Diseases: A Neurobiological View. Beijing: TsingHua University Press; 2010:32-80.

6. Mi H. The Systematic Classic of Acupuncture and Moxibustion: Huang-Ti Chen Chiu Chia I Ching (Jia Yi Jing). Boulder, CO: Blue Poppy Press; 1993.

7. Cheng X. Chinese Acupuncture and Moxibustion, rev. ed. Beijing: Foreign Languages Press; 1999.

8. Arakawa T, Sekiya S, Kumaki K, Terashima T. Ramification pattern of the deep branch of the lateral plantar nerve in the human foot. Ann Anat. 2005;187(3):287-296.

9. Shaw V. Chōng meridian: An ancient Chinese description of the vascular system? Acupunct Med. 2014;32(3):279-285.

10. Choi A, Kwon NY, Kim K, Kim Y, Oh J, Oh HM, Park JH. Anatomical localization of motor points of the abductor hallucis muscle: A cadaveric study. Ann Rehabil Med. 2017; 41(4):589-594.

11. Del Toro DR, Park TA. Abductor hallucis false motor points: Electrophysiologic mapping and cadaveric dissection. Muscle Nerve. 1996;19(9):1138-1143.

12. Fiolkowski P, Brunt D, Bishop M, Woo R, Horodyski M. Intrinsic pedal musculature support of the medial longitudinal arch: An electromyography study. J Foot Ankle Surg. 2003; 42(6):327-333.

13. Headlee DL, Leonard JL, Hart JM, Ingersoll CD, Hertel J. Fatigue of the plantar intrinsic foot muscles increases navicular drop. J Electromyogr Kinesiol. 2008;18(3):420-425.

14. Arinci Incel N, Genç H, Erdem HR, Yorgancioglu ZR. Muscle imbalance in hallux valgus: An electromyographic study. Am J Phys Med Rehabil. 2003;82(5):345-349.

15. Torres ALG, Ferreira MC. Study of the anatomy of the tibial nerve and its branches in the distal medial leg. Acta Ortop Bras. 2012;20(3):157-164.
16. Thordarson DB, Shean CJ. Nerve and tendon lacerations about the foot and ankle. J Am Acad Orthop Surg. 2005;13(3): 186-196.

17. Antoniadis G, Scheglmann K. Posterior tarsal tunnel syndrome: Diagnosis and treatment. Dtsch Arztebl Int. 2008; 105(45):776-781.

18. McSweeney SC, Cichero M. Tarsal tunnel syndrome-a narrative literature review. Foot (Edinb). 2015;25(4):244250.

19. Chundru U, Liebeskind A, Seidelmann F, Fogel J, Franklin P, Beltran J. Plantar fasciitis and calcaneal spur formation are associated with abductor digiti minimi atrophy on MRI of the foot. Skeletal Radiol. 2008;37(6):505-510.

20. Bjur D, Alfredson H, Forsgren S. The innervation pattern of the human Achilles tendon: studies of the normal and tendinosis tendon with markers for general and sensory innervation. Cell Tissue Res. 2005;320(1):201-206.

21. Dellon AL, Höke A, Williams EH, Williams CG, Zhang Z, Rosson GD. The sympathetic innervation of the human foot. Plast Reconstr Surg. 2012;129(4):905-909.

22. Staskin DR, Peters KM, MacDiarmid S, Shore N, de Groat WC. Percutaneous tibial nerve stimulation: A clinically and cost effective addition to the overactive bladder algorithm of care. Curr Urol Rep. 2012;13(5):327-334.

23. Tai C, Shen B, Chen M, Wang J, Roppolo JR, de Groat WC. Prolonged poststimulation inhibition of bladder activity induced by tibial nerve stimulation in cats. Am J Physiol Renal Physiol. 2011;300(2):F385-F392.

Address correspondence to:

Poney Chiang, PhD, RAc

Faculty of Health

School of Kinesiology and Health Sciences

Norman Bethune College

York University

4700 Keele Street, Room 341

Toronto, Ontario M3J 1 P3

Canada

E-mail: poney.chiang@riverclinic.ca 


\section{To receive CME credit, you must complete the quiz \\ online at: www.medicalacupuncture.org/cme}

\section{CME Quiz Questions}

Article learning objectives: After studying this article, participants should be able to identify the precise neuroanatomical structures that correlate with the acupuncture points termed kidney 1 to kidney 8 , and describe and cross-reference the precise neuroanatomical relationship with classical acupuncture point descriptions.

Publication date: February 7, 2019

Expiration date: December 31, 2019

\section{Disclosure Information:}

Authors have nothing to disclose.

Richard C. Niemtzow, MD, PhD, MPH, Editor-in-Chief, has nothing to disclose.

\section{Questions:}

1. Identify the incorrect statement:

In this article the authors:

a. Explore specific neuroanatomical structures associated with acupuncture points.

b. Propose that acupuncture point locations described in classical texts like the Zhen Jiu Jia Yi Jing (鍼炎甲乙經) serve fundamentally as coordinates to stimulate the peripheral nervous system.

c. Describe precise neuroanatomical "targets" for each acupuncture point discussed.

d. Describe precise neuroanatomical associations for points of the Foot Shao Yin channel.

e. Provide strong evidence that the classical acupoints are energetic entities distinct and separate from modern neuroanatomical structures.

2. Identify the incorrect statement:

The methods described by the authors include:

a. Contemporary standardized textbook locations from Cheng Xinnong's Chinese Acupuncture and Moxibustion (Foreign Languages Press).

b. Current anatomical literature.

c. Cadaveric dissection and research.

d. Meditation and 'inner-visioning' of energy pathways with the assistance of Nei Gong cultivation techniques.

e. Electrostimulation of acupuncture points in healthy volunteers.

3. Identify the incorrect statement:

a. Modern anatomical research has demonstrated that nearly all acupuncture points are associated with peripheral cutaneous nerves or deep nerve trunks.

b. The Zhen Jiu Jia Yi Jing (鍼炎甲乙經) or Systematic Classic on Acupuncture, written in 259 $\mathrm{AD}$, is considered to be the first complete acupuncture manual to detail the locations of acupuncture points and channels. c. Neuroanatomical targets were verified by electrostimulation on live, healthy subjects.

d. Ki-4, located on the medial calcaneal nerve branch which provides sensation to the heel pad may be a useful acupoint in the treatment of foot-related conditions.

e. The authors suggest that choice of points according to relevant neuroanatomy plays a secondary role to classically described point prescriptions.

4. Identify the incorrect statement:

The authors suggest:

a. Standardization of precise neuroanatomical targets has an importance for acupuncture research.

b. The reliance upon two-dimensional surface coordinates for acupoint stimulation in research may lead to inadvertent stimulation of different neuroanatomic structures within a research trial protocol.

c. Reliance upon two-dimensional surface descriptions for acupoints may lead to stimulation of different neuroanatomic structures even though the same acupuncture point (according to surface anatomy) is being needled.

d. Standardization of precise neuroanatomical targets for each acupuncture point may improve reproducibility of results in clinic and research.

e. Data collected on relevant neurohormonal responses associated with the neuroanatomical ones described in this paper will be reported in a future research paper.

5. Identify the incorrect statement:

a. KI-7 and KI-8 correspond to the posterior tibial neurovascular bundle.

b. The posterior tibial nerve is a mixed motor sensory nerve with axons that travel through L4-S3 spinal 
nerve roots associated with sensory and bladder control of the bladder.

c. KI-7 has a history of acupuncture use in China for bladder syndromes.

d. These authors report on studies demonstrating the use of KI-1, which corresponds to the deep branch of the lateral plantar nerve, for bladder syndromes.

e. In a cat model of posterior tibial nerve stimulation, researchers studied 5 and $30 \mathrm{~Hz}$ stimulation and demonstrated increased bladder capacity with some techniques of electrostimulation.

\section{Continuing Medical Education - Journal Based CME Objectives:}

Articles in Medical Acupuncture will focus on acupuncture research through controlled studies (comparative effectiveness or randomized trials); provide systematic reviews and meta-analysis of existing systematic reviews of acupuncture research and provide basic education on how to perform various types and styles of acupuncture. Participants in this journal-based CME activity should be able to demonstrate increased understanding of the material specific to the article featured and be able to apply relevant information to clinical practice.

\section{CME Credit}

You may earn CME credit by reading the CME-designated article in this issue of Medical Acupuncture and taking the quiz online. A score of $75 \%$ is required to receive CME credit. To complete the CME quiz online, go to http://www .medicalacupuncture.org/cme - AAMA members will need to login to their member account. Non-members have the opportunity to participate for a small fee.

Accreditation: The American Academy of Medical Acupuncture is accredited by the Accreditation Council for Continuing Medical Education (ACCME).

Designation: The AAMA designates this journal-based CME activity for a maximum of 1 AMA PRA Category 1 Credit $^{\mathrm{TM}}$. Physicians should claim only the credit commensurate with the extent of their participation in the activity. 